\title{
Influence of a Controlling Factor on the Unsteady Viscosity Profile of Deoxyhemoglobins
}

\author{
S. Moussiliou ${ }^{1}$, S. Massou ${ }^{1} \&$ A. Essoun ${ }^{1}$ \\ ${ }^{1}$ Département de physique, Faculté des Sciences et Technique, Université d'Abomey-Calavi, 06 BP. 48 Cotonou, \\ République du Bénin \\ Correspondence: S. Moussiliou, Département de physique, Faculté des Sciences et Technique, Université \\ d'Abomey-Calavi, 06 BP. 48 Cotonou, République du Bénin. E-mail: moussiliousikirou@yahoo.fr
}

Received: June 5, 2012 Accepted: June 23, 2012 Online Published: September 17, 2012

doi:10.5539/jmsr.v1n4p79 URL: http://dx.doi.org/10.5539/jmsr.v1n4p79

\begin{abstract}
The mechanism of polymerization is linking to the structural viscosity $\eta^{*}(t, x)$ which depends on the time $\mathrm{t}$ and a controlling parameter $x$. The controlling factor which influences the aggregation dynamics can be limited to, say, the volume $\phi$ function, the temperature $T$, the déoxy-HbS, concentration $c$ and obviously a constant shear rate $\dot{\gamma}$. The mathematical model for $\eta^{*}(t, x)$ need a sub-model for each controlling factor $x$. This constraint drives to derive a rheological model $\eta^{*}(t, x)$ can be directly taken into account in the parameters base.

In the present work, we study the influence of these controlling factors on the unsteady viscosity profiles obtained during the time course of aggregation process.
\end{abstract}

Keywords: deoxyhemoglobin $S$ aggregation, rheological model, unsteady viscosity profiles, influence of a controlling factor

\section{Introduction}

During the last three decades, flow properties of dispersions have been a subject of increasing interest owing to the great variety of such systems found in nature and industry, especially in the case of highly concentrated suspensions of colloidal particles as solutions of deoxyhemoglobin $S$.

More precisely, the solution-gel transformation of sickle hemoglobin was first described (Harris \& Bensusan, 1980; Hofrichter, Ross, \& Easton, 1974) and it was proposed that molecular aggregation combined with orientation of the aggregates formed the basis of the erythrocyte sickling phenomenon (Krieger \& Dougherty, 1959). Only very recently attempts have been made to investigate the kinetics of this transformation by various technics (de Kruif, van Lersel, \& Vrij, 1985; Quemada \& Droz, 1983; Quemada, 1984, 1998a, 1998b; Reynolds, 1886).

In the studies reported by (Harris \& Bensusan, 1980), on a time-dependent change in the specific viscosity of purified deoxyhemoglobin $S$ solutions, we observed a latent period with no detectable increase in viscosity, followed by a rapid increase in viscosity to gel formation. The very marked influence that the shear rate $\dot{\gamma}$ has upon the duration of the lag phase is illustrated in Figure (1) reported by (Harris \& Bensusan, 1980).

The controlling factor which influences the aggregation dynamics can be limited to, say, the volume $\phi$ function, the temperature $T$, the déoxy-HbS, concentration $c$ and obviously a constant shear rate $\dot{\gamma}$.

The purpose of this work is to compare the profiles obtained by the influence of controlling factor with results of (Harris \& Bensusan, 1980).

\subsection{Main Feature of the Rheological Model}

The effective structural viscosity $\eta^{*}(t, x)$ which depends on time and a dimensionless controlling parameter $x$ is an important parameter which account for the mechanism of the polymerization. The controlling factor which influences the aggregation dynamics can be limited to, say, the volume function $\phi$, the temperature $T$, the déoxy-HbS concentration, and obviously a constant shear rate $\dot{\gamma}$.

In the sequel, for the sake of simplicity we introduce a dimensionless controlling factor $x=x / x_{c}$, where $x_{c}$ is a characteristic value of the variable $x$. 
The elaboration of a rheological model for which is more relevant because each of these controlling factors can be directly taken into account in the parameters base $\eta^{*}(t, \bar{x})$ The analytical function $\eta^{*}(t, \bar{x})$ which is suitable for data fitting reads:

$$
\eta^{*}(t, \quad \bar{x})=K[1+\alpha \exp (-c t)] /[1+\operatorname{bexp}(-\mathrm{ct})]^{\mathrm{n}}
$$

with the following definitions:

$$
\left\{\begin{array}{c}
a=\xi \exp \left(\omega t_{d}\right) \\
b=\vartheta \exp \left(\omega t_{d}\right) \\
c=\omega \\
K=h \eta(\vec{x}) \delta(\bar{x})
\end{array}\right.
$$

Where

$\omega$ is the frequency of relaxation,

$t_{d}$ is delay time,

$\eta$ is positive constant.

The constants $a, b$, and $K$ depend on the steady structural viscosity $\eta(\bar{x})$.

Obviously, the constant $K$ has the unit of a viscosity. Moreover, this limiting value $K=\lim _{t \rightarrow \infty} \eta^{*}(t, \bar{x})=\eta^{* \infty}$ can be directly measured on the tracing.

It is interesting to observe that (1.1) and (1.2) has the same analytical forms. However, as we can see, the present derivation is more relevant for rheological studies.

However, the detailed functional form of the steady viscosity $\eta(\bar{x})$ is still a problem to be determined from either theory or experiment. In this case various theoretical approaches have been proposed. Particularly (Dejardin \& Olatunji, 1985) obtained the following relationship:

$$
\eta(\bar{x})=\eta_{\infty}\left[1-(1-\chi) \lambda_{e q}(\bar{x})\right]^{-n}
$$

Where

$$
\lambda_{e q}(\overline{\boldsymbol{x}})=\frac{1}{\exp (\overline{\mathbf{x}})}
$$

is the equilibrium value of the structural parameter that depends on the dimensionless controlling factor $x=x / x_{c}$, where $x_{c}$ is a characteristic value of the variable $x$.

$$
\chi=\left(\frac{\eta_{\infty}}{\eta_{0}}\right)^{\frac{1}{n}}
$$

Expanding $\exp (\bar{x})$ in a Taylor series limited to the first order term we get:

$$
\lambda_{e q}(\overline{\boldsymbol{x}})=\frac{1}{1+\overline{\mathbf{x}}}
$$

In $(1.2) \delta(\bar{x})$ is the coefficient of visco-elasticity readily defined by:

$$
\delta(\overline{\boldsymbol{x}})=\left[1+\frac{4 \mathrm{p} \rho \mathrm{G}(\overline{\mathrm{x}})}{\eta(\overline{\mathrm{x}})^{2}}\right]^{1 / 2}
$$

where the structural elastic modulus $\mathrm{G}(\bar{x})$ is given by the following relationships:

$$
G(\bar{x})=G_{\infty}\left[1-(1-\kappa) \lambda_{e q}(\bar{x})\right]^{-m}
$$

with

$$
\kappa=\left(\frac{\eta_{\infty}}{\eta_{0}}\right)^{\frac{1}{m}}
$$




\section{Influence of a Controlling Factor on the Unsteady Viscosity Profile}

\subsection{Influence of the Concentration of the Deoxy-HbS}

* Method

If $\bar{x}=s=c / c_{e}$ is the supersaturation ratio introduced by (Hofrichter, Ross, \& Easton, 1974) in the aggregation kinetics, then the steady state viscosity is given by (1.3), viz :

$$
\eta(s)=\eta_{\infty}\left[1-(1-\chi) \lambda_{e q}(s)\right]^{-n}
$$

Then we have $\frac{\partial \eta(s)}{\partial(s)}<0$ for $\chi<1$.

It is interesting to observe that this theoretical result is in good agreement with experimental data reported by (Harris \& Bensusan, 1980).

According to (1.3), the delay time $t_{d}(s)$ on the effective viscosity profiles $\eta^{*}(t, S)$ can be written as:

$$
t_{d}(s)=t_{\infty}\left[1-(1-\mu) \lambda_{e q}(s)\right]^{-q}
$$

where $\lambda_{e q}(s)$ is given by (1.3) or (1.6).

As can be readily seen, (2.2) shows that the delay time $t_{d}(s)$ decreases when the concentration of the deoxy-HbS increases. Such a theoretical result agrees with the experimental data.

More precisely, taking into account the variations of $t_{d}(s)$ and $\eta(s)$ which are the characteristics parameters in the (1.1) that define the viscosity $\eta^{*}(t, s)$ we obviously observe that this function decreases when the concentration $c$ increases.

* Qualitative Numerical Calculations

For illustration, we used (1.1) to calculate three viscosity profiles with the following values of the concentration $c$.

$$
c=25 \mathrm{mg} / \mathrm{l}, c=45 \mathrm{mg} / \mathrm{l} \text {, and } c=55 \mathrm{mg} / \mathrm{l}
$$

As show in Figure (1) it is interesting to observe that the theoretical profiles faithfully reproduce the main characteristic of the experimental results qualitatively.

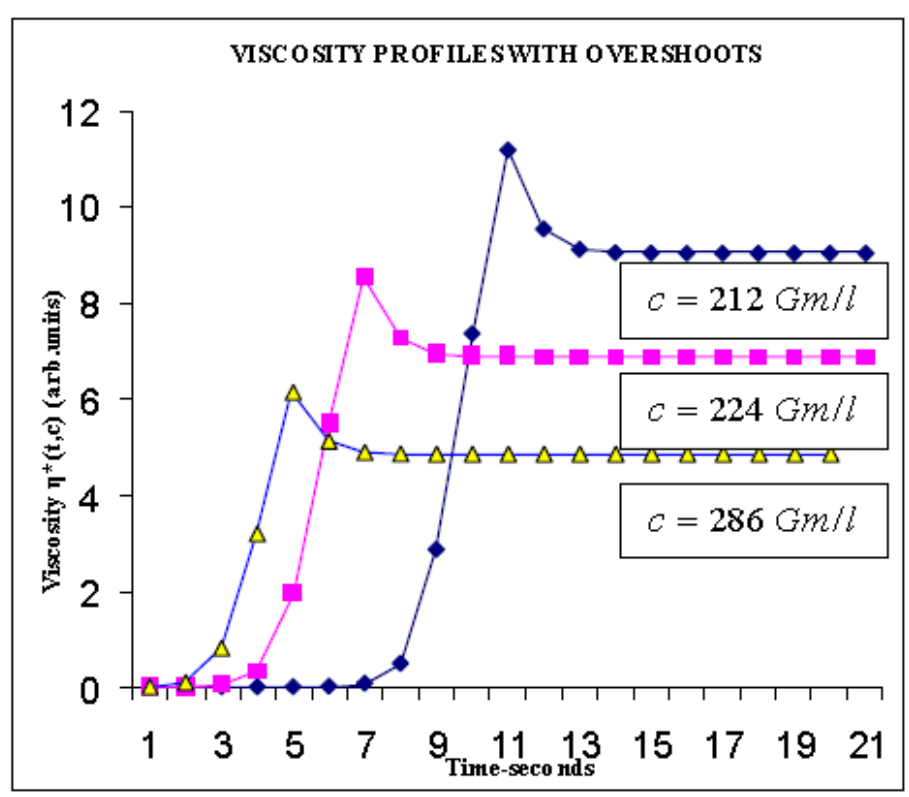

Figure 1. Influence of the concentration and the viscosity profiles 


\subsection{Influence of the Shear Rate $\dot{\gamma}$, the Volume Fraction $\phi$, and the Temperature T}

As indicated, we have found that for the others controlling factors, the study may be rationalized by introducing in the parameters base, the dimensionless shear rate defined by the well-known translational Péclet number, viz:

$$
\bar{x}=\theta(\dot{\gamma}, \boldsymbol{\phi}, \boldsymbol{T})=\frac{6 \pi \eta r^{3} \dot{\gamma}}{\kappa_{B} T}=P_{e}
$$

which varies with the solvent viscosity $\eta_{F}$, the constant shear rate $\dot{\gamma}$, the particles radius $r$, the thermal energy $k_{B} T$, where $k_{B}$ is the Boltzmann constant and $T$ the absolute temperature.

It is interesting to observe that (2.3) can be simply evaluated from kinetic equation for aggregation $\leftrightarrows$ desegregation of structural units viz:

$$
\frac{d \lambda}{d t}=\frac{1-\lambda}{\tau_{A}}-\frac{\lambda}{\tau_{D}}
$$

where, $\tau_{A}$ and $\tau_{D}$ are the times characterizing the formation and rupture of the aggregates respectively. More generally, these times are associated to either organization or disorganization of the structure, and consequently depend on the shear rate $\dot{\gamma}$.

In steady state condition $d \lambda / d t$, the steady value of the structural parameter $\lambda=\lambda_{\text {eq }}(\dot{\gamma})$ will be from (2.4):

$$
\lambda_{e q}(\dot{\gamma})=\frac{1}{1+\theta(\dot{\gamma})}
$$

where

$$
\theta(\dot{\gamma})=\tau_{A} / \tau_{D}
$$

$\tau$ being a characteristic time. In (1.9) and (2.3) the parameter $\omega$ is a shear dependent frequency of the structure which is a symmetrical relation in $\tau_{A}$ and $\tau_{D}$, viz (Goldsmith \& Mason, 1916; Krieger \& Dougherty, 1959):

$$
\omega=\frac{\tau_{A}+\tau_{D}}{\tau_{A} \tau_{D}}
$$

It is interesting to observe that (2.5) obtained by (de Kruif, van Lersel, \& Vrij, 1985) is exactly our (1.6) with $\exp (\bar{x})=\theta(\gamma)$. More precisely as indicated, we obtain (2.5) using (1.4), and expanding $\exp (\bar{x})$ in a Taylor series limited to the first order term.

However, it is worthy of note that our nonlinear dynamic model is self- consistent because no particular kinetic equation for the structural variable is used as sub-model (Dejardin \& Olatunji, 1985), $\lambda(x, t)$ is used as sub-model (Dejardin \& Olatunji, 1985), and the steady value given by (1.4) comes directly from the theory.

Moreover, from (1.4) and (1.6) we obtained as required $\lambda_{e q}(0)=1$, for complete building up, or $\lambda_{e q}(\infty)=0$ for complete breakdown of the structure respectively.

Now, it is more relevant to give a phenomenological foundation of (2.3). For this purpose, $\tau_{A}$ can be considered as a constant, which is proportional to the Brownian diffusion time for colloidal suspensions of spherical particules of radius $r$.

This characteristic time is given by (Smoluchowski, 1916) :

$$
\tau_{A}=\beta \frac{\eta F \gamma^{3}}{k_{B} T}
$$

with $\beta=6 \pi$ or $\beta=8 \pi$, for translational or rotational diffusion respectively.

According to (Goldsmith \& Mason, 1916), the time $\tau_{D}$ can be taken as:

$$
\tau_{D}=\dot{\gamma}^{-1}
$$

Hence, using (2.1) and (2.2) in (1.9), we obtain as desired the translational Péclet number (1.6).

For practical purpose, it is useful to introduce in (2.3) instead of the particles radius $r$, the volume fraction $\phi$ :

$$
\phi=4 \pi r^{3} n / 3
$$

where $n$ is the number density. Hence, we get: 


$$
\theta(\dot{\gamma}, \boldsymbol{\phi}, \boldsymbol{T})=\frac{9 \eta_{F} \phi \dot{\gamma}}{2 n \kappa_{B} T}
$$

Consequently, (1.3), (1.4) and (1.6) become:

$$
\mu=\mu_{\infty}\left[1-(1-\chi) \lambda_{e q}(\dot{\gamma}, \boldsymbol{\phi}, \boldsymbol{T})\right]^{-n}
$$

Where

$$
\lambda_{e q}(\dot{\gamma}, \boldsymbol{T})=\frac{1}{\operatorname{Exp}[\theta(\dot{\gamma}, \boldsymbol{\phi}, \boldsymbol{T})]}
$$

consequently, according to (1.6) we have :

$$
\lambda_{e q}(\dot{\gamma}, \boldsymbol{\phi}, \boldsymbol{T})=\frac{1}{1+\theta(\dot{\gamma}, \boldsymbol{\phi}, \boldsymbol{T})}
$$

Furthermore it is worthy to note that by linearization $(n=-1)$, the general equation (2.12) directly leads to (Krieger \& Dougherty, 1959) equation, viz:

$$
\eta=\eta_{\infty}+\left(\eta_{0}-\eta_{\infty}\right) \lambda_{e q}(\dot{\gamma}, \boldsymbol{\phi}, \boldsymbol{T})
$$

The relation (2.15) shows that for $\eta_{0}>\eta_{\infty}$, the steady viscosity decreases when the temperature increases, in accordance experimental data and generally fit by phenomenological equations. For detail see (Briant, 1956). Among these phenomenological equations let mentioned the equation given by (Andrade $\&$ da, 1934) .

$$
\eta=\operatorname{aexp}\left(\frac{b}{T}\right)
$$

provides that the temperature $T$ is different from zero, $a$ and $b$ being positive constants.

The second example is the equation giving by (Reynolds, 1886):

$$
\eta=\operatorname{aexp}(-\beta T)
$$

where $\alpha$ and $\beta$ are two positive constants.

However, at this stage of modeling, it is worthy of note that our general equation (2.12), also gives result in excellent agreement with experimental data. For this purpose, if the controlling factor is the temperature, the steady viscosity can be written in the following form:

$$
\eta(T)=\eta_{\infty}[1-(1-\chi) \exp (-T c / T)]^{-n}
$$

provides that the temperature is different from zero, $T_{c}$ being a characteristic temperature After little algebra, we obtain:

$$
\frac{d \eta(T)}{d T}=-n(\chi-1) \frac{T_{C}}{T^{2}} \frac{\eta(T) \exp \left(-T_{C} / T\right)}{1+(\chi-1) \exp \left(-T_{C} / T\right)}
$$

Hence, it is readily seen from (2.19) that for $\chi>1$, the structural viscosity (2.18) also decreases when the temperature increases. We display in Figure (2) the graphs of the three viscosity models (2.16), (2.17) and (2.18).

For the viscosity $\mu(\phi)$ versus volume fraction $\phi$ relationship, (1.2) leads to a new $\mu(\phi)$. 


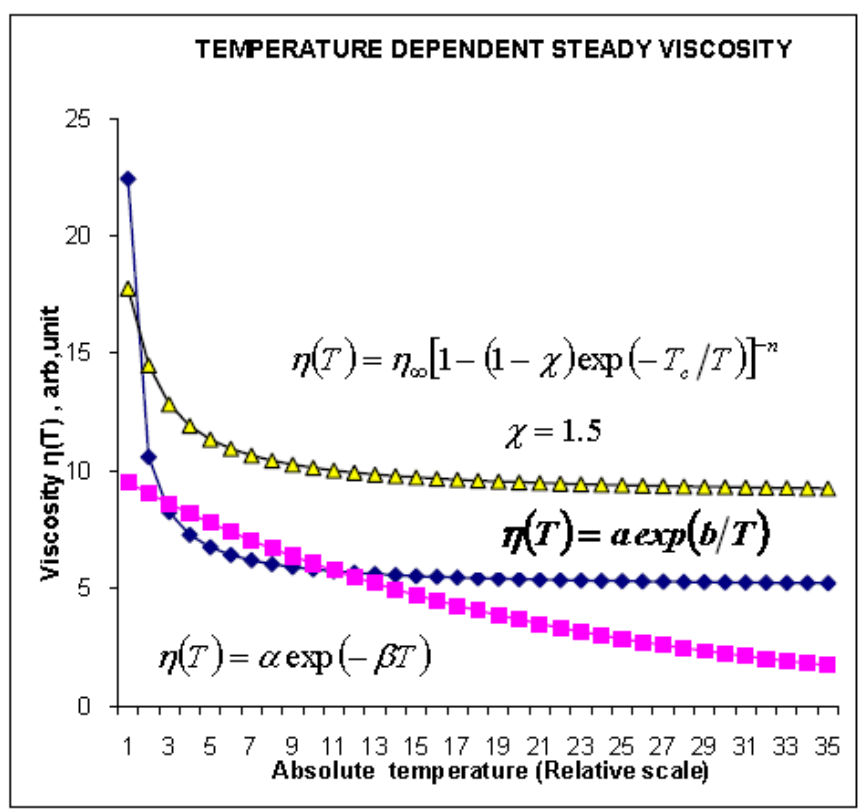

Figure 2. Temperature dependent models of viscosity

\subsubsection{Influence of the Shear Rate $\dot{\gamma}$}

\section{* Method}

To take into account the influence of the shear rate $\dot{\gamma}$, on the unsteady viscosity profiles, one may keep $\phi$ and $\boldsymbol{T}$ unchanged in (2.5) which for this purpose is written in the

suitable form:

$$
\bar{x}=\theta(\dot{\gamma})=\frac{\dot{\gamma}}{\dot{\gamma}_{c}} \quad \dot{\gamma}_{c}=\frac{2 n k_{B} T}{9 \eta_{F} \phi}
$$

$\dot{\gamma}_{c}$ being a characteristic shear rate.

* Qualitative Numerical Calculations

The experimental viscosity profiles obtained by (Harris \& Bensusan, 1980) are reported in Figure (4).

For illustration, we used (1.1) to calculate three viscosity profiles with the following values of the shear rate $\dot{\gamma}$ :

$$
\dot{\gamma}=10 \sec ^{-1}, \dot{\gamma}=20 \sec ^{-1} \text { and } \dot{\gamma}=30 \sec ^{-1}
$$

As show in Figure 2 it is interesting to observe that the theoretical profiles faithfully reproduce the main characteristic of the experimental results qualitatively: Figure 3.

More precisely:

a) the delay time $t_{d}(\dot{\gamma})$ decreases when the shear rate $\dot{\gamma}$ increases.

b) the maximum of the viscosity profiles (overshoot) decreases when the shear rate $\dot{\gamma}$ increases.

c) The maximum change in viscosity decreases when the shear rate $\dot{\gamma}$ increases.

* Experimental Data Fitting

Based on the time-dependent viscosity values reported by (Goldsmith \& Mason, 1916), the mode parameters ( $a$, $b, c, n)$ of (2.22) are computed using non linear square method.

The results are presented in Figures (3) and Figure (4). As can be seen, the analytical function $\eta^{*}(t, \dot{\gamma})$ satisfactorily fit the experimental data.

Consequently, as indicated, we observe that our theoretical model clearly improves on the sufficient and necessary conditions for any good model of interpretation (Quemada \& Droz, 1983), viz: 
- he contains a minimum number of parameters

- he gives a good data fitting

- the parameters can be readily calculated

- the parameters have physical significance

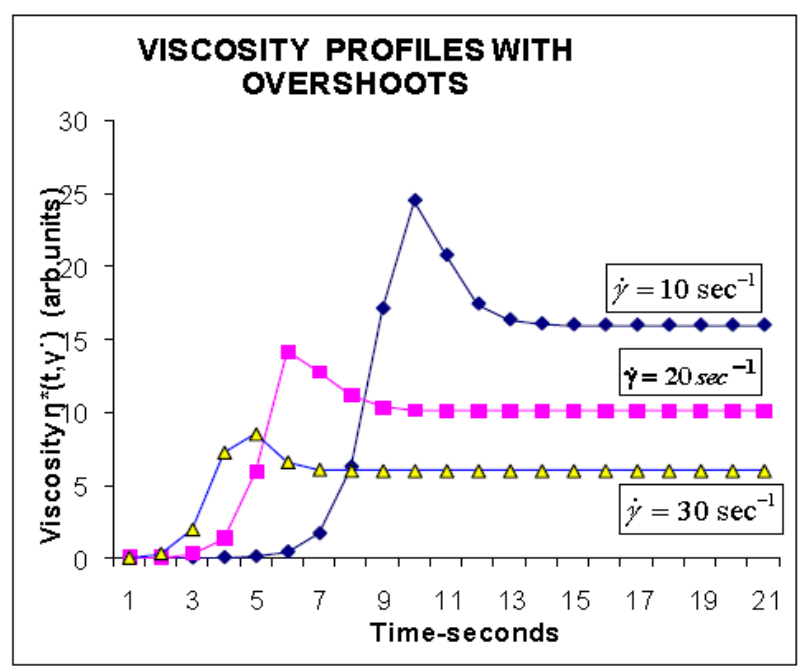

Figure 3. Influence of shear rate and the viscosity profiles (Goldsmith \& Mason, 1916)

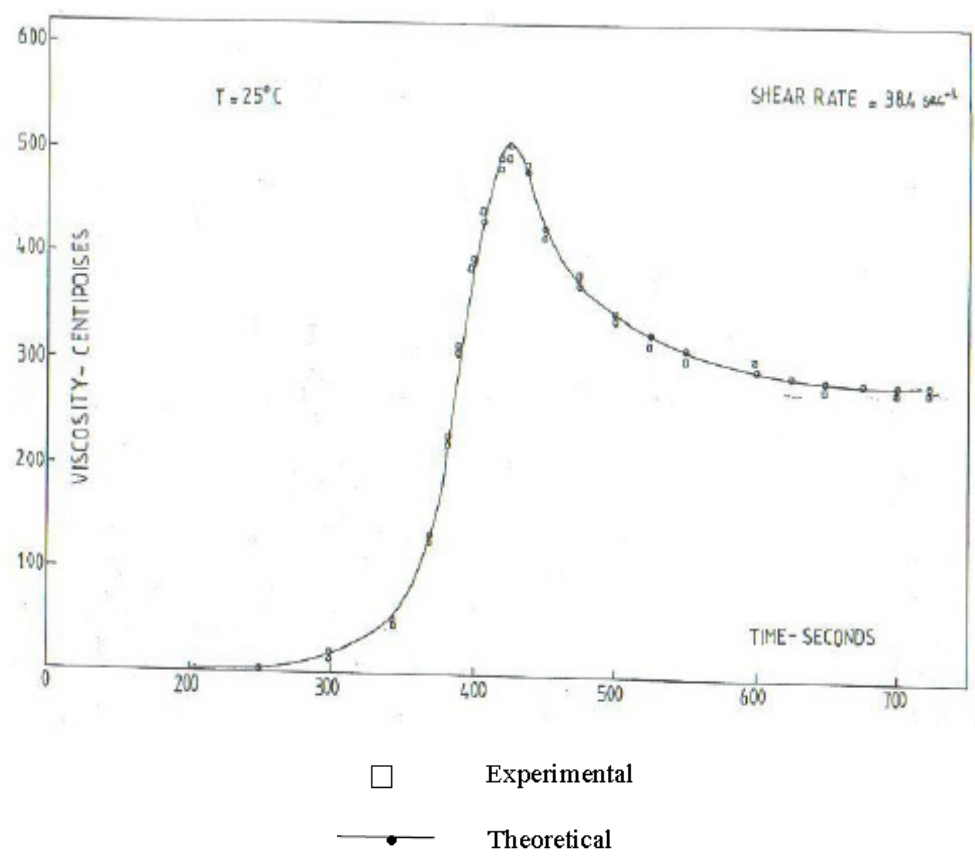

Figure 4. Viscosity profile-data fitting (Goldsmith \& Mason, 1916)

\subsubsection{Influence of the Volume Fraction $\phi$}

* Method

To take into account the influence of the volume fraction $\phi$ on the unsteady viscosity. profiles, one may keep $\dot{\gamma}$ and $\boldsymbol{T}$ unchanged in (2.5) which for this purpose is written in the suitable form:

$$
\bar{x}=\theta(\phi)=\frac{\phi}{\phi_{c}} \quad \phi_{c}=\frac{2 n k_{B} T}{9 \eta_{F} \dot{\gamma}}
$$


$\phi_{c}$ being a characteristic volume fraction.

* Qualitative numerical calculations

For illustration, we used (1.1) to calculate three viscosity profiles with the following values of the volume fraction $\phi_{c}$

$$
\phi=0.25, \phi=0.35 \text { and } \phi=0.45
$$

The equilibrium values are respectively:

$$
\phi=0.74, \phi=0.67 \text { and } \phi=0.61
$$

As show in Figure (5) it is interesting to observe that the theoretical profiles faithfully reproduce the main characteristic of the experimental results qualitatively.

2.2.3 Influence of the Temperature $T$

* Method

To take into account the influence of the temperature $T$ on the unsteady viscosity, profiles, one may keep $\gamma$ and $\phi$ unchanged in (2.5) which for this purpose is written in the suitable

form:

$$
\bar{x}=\theta(T)=\frac{T_{C}}{T} \quad T_{c}=\frac{2 n k_{B}}{9 \eta_{F} \dot{\gamma} \phi}
$$

$T c$ being a characteristic temperature which is now well defined.

* Qualitative numerical calculations

For illustration, we used (1.1) to calculate three viscosity profiles with the following values of the temperature :

$$
T=289.2 \mathrm{~K}, T=299.2 \mathrm{~K} \text { and } T=309.2 \mathrm{~K}
$$

The equilibrium values are respectively:

$$
T_{\text {eq } 1}=0.4762 \mathrm{~K}, T_{\text {eq } 2}=0.4847 \mathrm{~K} \text { and } T_{\text {eq } 3}=0.4929 \mathrm{~K}
$$

The critical absolute temperature is: $T_{c}=318 \mathrm{~K}$.

As show in Figure (6) it is interesting to observe that the theoretical profiles faithfully reproduce the main characteristic of the experimental results qualitatively.

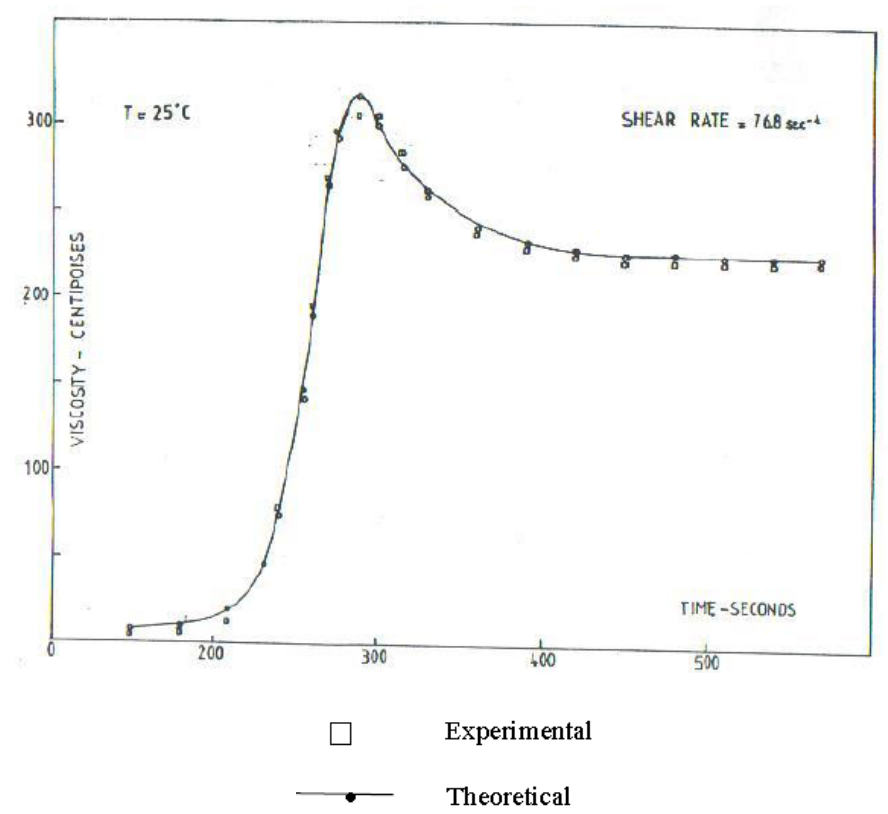

Figure 5. Viscosity profile-data fitting 


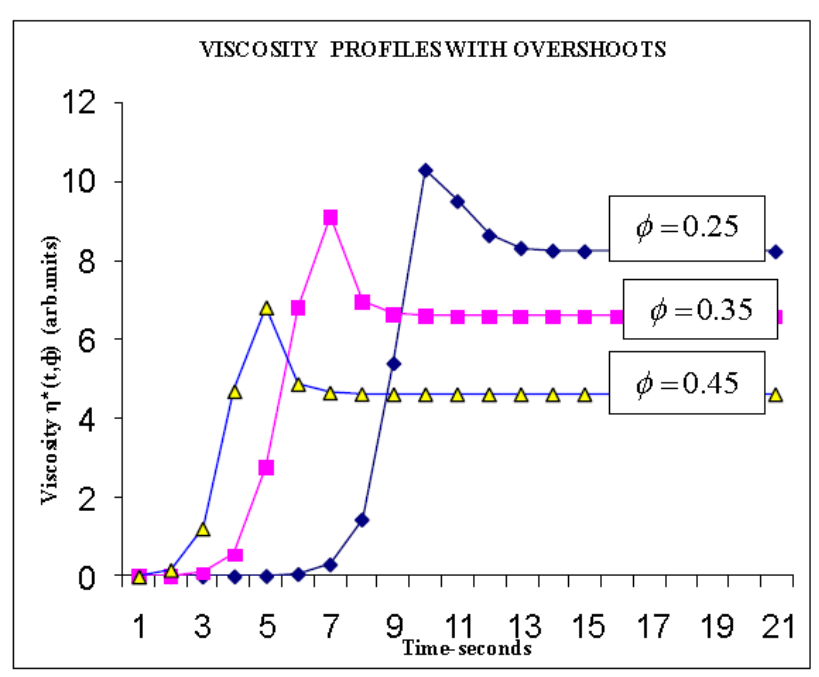

Figure 6. Influence of the volume fraction and the viscosity profiles

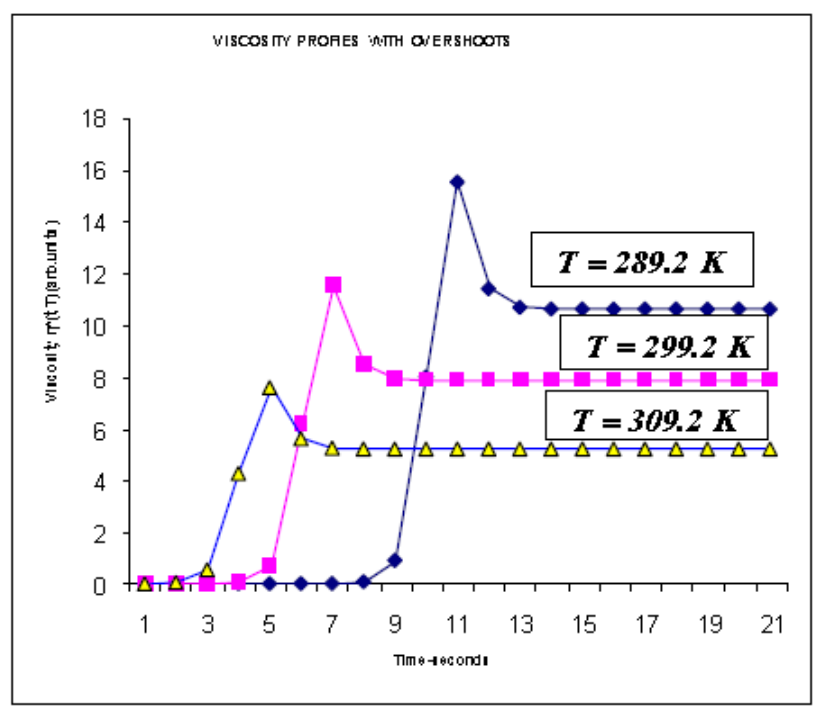

Figure 7. Influence of temperature and the viscosity profiles

\section{Conclusion}

The effective structural viscosity $\eta^{*}(t, x)$ which depends on time and a controlling parameterx is an important parameter $x$ which account for the mechanism of the polymerization. The controlling factor which influences the aggregation dynamics can be limited to, say, the volume function $\phi$, the hematocrit $H$, the temperature $T$, the deoxyhemoglobin $S$, concentration and obviously a constant shear rate $\dot{\gamma}$. Because the limited applicability of the previous mathematical model derived by (Dejardin \& Olatunji, 1985) which need a sub-model for each controlling factor on unsteady viscosity profiles obtained during the time course of aggregation process.

Consequently, when the controlling factor $x$ is the shear rate $\dot{\gamma}=\dot{\gamma}\left(t_{c}\right)$ where $t_{c}$ is the duration of the perturbations of the system at equilibrium. Our theoretical results are in agreement with the experimental data of (Goldsmith \& Mason, 1916).

However, because the limited applicability of this mathematical model which need a sub-model for each controlling factor $\mathrm{x}$, the rheological model derived for $\eta^{*}(t, x)$ is more relevant because each of these controlling factor can be directly taken into account in the parameters base. Moreover, it is worthy to note that the mathematical model and the rheological model lead for $\eta^{*}(t, x)$ the same analytical form. For practical purpose, and by way of illustration, we study, the effects induced by these controlling factors on the viscosity profiles. 
The theoretical profiles faithfully reproduce the main characteristics of the experimental results reported by (Goldsmith \& Mason, 1916).

\section{References}

Andrade, E. N. \& da C. (1934). Theory of viscosity of liquids. Phil. Mag., 17, 497- 698.

Briant, J. (1956). Etude des propriétés rhéologiques des graisses à l'aide du viscosimètre SOD. Rev. Inst. Franç. Du Pétrole, 11, 113-287.

de Kruif, C. G., van Lersel, E. M. F., \& Vrij, A. (1985). Hard sphere colloidal Dispersions-Viscosity as a function of shear rate and volume fraction Dispersions-Viscosity as a function of shear rate and volume fraction. J. Chem. Phys., 83, 4718-4725. http://dx.doi.org/10.1063/1.448997

Dejardin, J. L., \& Olatunji, L. (1985). Mathematical model of deoxyhemoglobin S aggregation kinetics. Journal de biophysique et de biomécanique, 9(2), 75-79.

Goldsmith, \& Mason. (1916). Koloid. Z., 190-195.

Harris, J. W., \& Bensusan, H. B. (1980). The kinetics of solution-gel transformation of deoxyhemoglobin S by continuous monitoring of viscosity. J. Lab. Clint. Med., 86, 564.

Hofrichter, J., Ross, P. D., \& Easton, W. A. (1974). Kinetics and mechanism of Deoxy- hemoglobin S GelationA new approach to understanding sickles cell disease. USA: Proc. Natl. Acad. Sci., 71, 48-64. http://dx.doi.org/10.1073/pnas.71.12.4864

Krieger, I. M., \& Dougherty, T. J. (1959). A mechanism for non-newtonian flow in suspensions of rigid spheres. Trans. Soc. Rheol., 3, 137-152. http://dx.doi.org/10.1122/1.548848

Quemada, D. (1984). Towards a unified model of elasto-thixotropy of biofluid. Biorheology, 21, 423-436.

Quemada, D. (1998a). Rheological modelling of complex fluid. I: The concept of effective volume fraction revisited. The European Physical Journal Applied Physics, 119-127. http://dx.doi.org/10.1051/epjap:1998125

Quemada, D. (1998b). Rheological modelling of complex fluid. II: Shear thickening behaviour due to shear induced flocculation. The European Physical Journal Applied Physics, 175-181. http://dx.doi.org/10.051/epjap:1998170

Quemada, D., \& Droz, R. (1983). Blood viscoelasticity and thixotropy from stress formation and relaxation measurements; A unified model. Biorheology, 20, 635-651.

Reynolds, O. (1886). On the theory of lubrication and its application to Mr. B. Tower's experiments, including and experimental determination of the viscosity of olive oil. Phil. Trans., 177A, 157-234. http://dx.doi.org/10.1098/rstl.1886.0005

Smoluchowski, M. (1916). Koloid. Z, 190-195. 